\title{
PHOTOGRAMMETRIC VERIFICATION OF FIBER OPTIC SHAPE SENSORS ON FLEXIBLE AEROSPACE STRUCTURES
}

\author{
Jason P. Moore, Matthew D. Rogge, Thomas W. Jones \\ NASA Langley Research Center \\ Hampton, $V A$
}

\section{Introduction}

Multi-core fiber (MCF) optic shape sensing offers the possibility of providing in-flight shape measurements of highly flexible aerospace structures and control surfaces for such purposes as gust load alleviation, flutter suppression, general flight control and structural health monitoring. Photogrammetric measurements of surface mounted MCF shape sensing cable can be used to quantify the MCF installation path and verify measurement methods.

\section{Multi-core Fiber Optic Shape Sensing}

MCF shape sensing is accomplished in the simplest form via converting co-located fiber Bragg gratings (FBG's) into local bend measurements and constructing overall fiber shape through the conjunction of successive local bend measurements [1]. In an effort to improve accuracy, more sophisticated methods of utilizing MCF FBG measurements have been developed [2] and a method of using Rayleigh scatter signatures of MCF to determine cable shape has also been disclosed [3]. The example subsequently discussed assumes the use of the Frenet-Serret geometric solution [2] to determine the shape of a three-core fiber with co-located FBG's located at $1 \mathrm{~cm}$ intervals along the fiber. The first step is to measure the strain of each core's FBG triplets using a distributed system such as an Optical Frequency Domain Reflectometer (OFDR) [4]. The FBG strain measurements are referenced to a baseline recorded while the fiber is held as straight as possible with zero strain. The measurements are then used to derive length-dependent local curvature and bend direction functions of the MCF. Lastly, those functions are included in a numerical solution of the Frenet-Serret equations to which the known global starting point of the MCF provides the initial conditions.

\section{Photogrammetry}

Photographic images are the result of a perspective projection of the three-dimensional (3D) object onto the two-dimensional (2D) image plane. Consequently, two or more photographic images can be reverse-engineered to derive the $3 \mathrm{D}$ shape or position of the original object. This process is called photogrammetry, and the solution provides a quantitative relationship between a $3 \mathrm{D}$ object and the $2 \mathrm{D}$ images acquired by the cameras. While photogrammetry has its roots in the topographic mapping and surveying field, the last decade has seen "close-range" photogrammetric techniques developed to support various

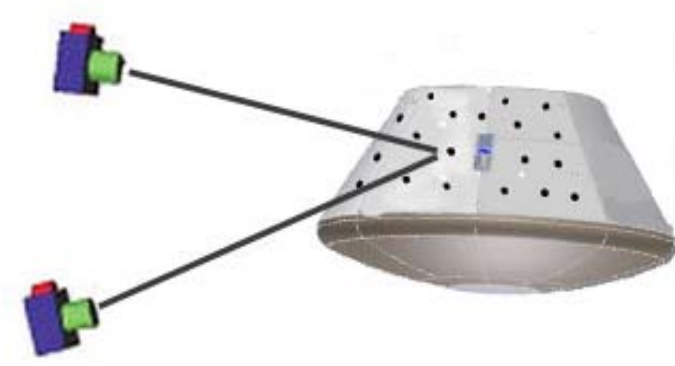

Figure 1: High contrast targets visible from multiple perspectives. industrial and research applications [5]. For example, in some areas of aeronautics aero-elastic experimentation (e.g., model deformation and wing twist) photogrammetric measurements have become part of the standard data set. Accurate photogrammetric measurements require the photography of high-contrast surface features that appear in at least two images (Figure 1). Because many objects of interest do not naturally exhibit such features, a common practice is to attach circular retroreflective targets to the object to artificially provide high-contrast features. When illuminated, these targets reflect light directly back to the illuminating source, causing the targets to appear very bright in relation to the background. 


\section{Application}

Temporarily applying retroreflective targets to a surface-mounted fiber and scanning the fiber with a photogrammetric system can provide independent measurements which can ultimately be used as a reference for assessing MCF shape sensing accuracy during testing and verification of a MCF shape sensing system. Figure 2 shows the shape measurement result from a $1 \mathrm{~m}$ MCF cable after installation on a flexible surface (blue) and after flexing of the same surface (red). Flexing profiles of the surface can prove hard to control and/or predict, as is the case of an experimental flexible control surface experiencing aero loads during flight testing. Prior to such testing, a photogrammetric system can provide the reference shape measurements of the control surface to which MCF shape measurements can be compared. The installation of a MCF shape sensing cable can be accomplished through bonding, taping, or insertion into pre-installed sleeving. While it is desirable to route the cable in a predetermined scheme onto the surface of interest, complying to exact layout specifications can be considerably difficult for large areas. Referring to blue shape profile of Figure 2, while the installation of the cable was performed as diligently as possible, the precise layout of the cable after installation can only be determined by an independent measurement technology

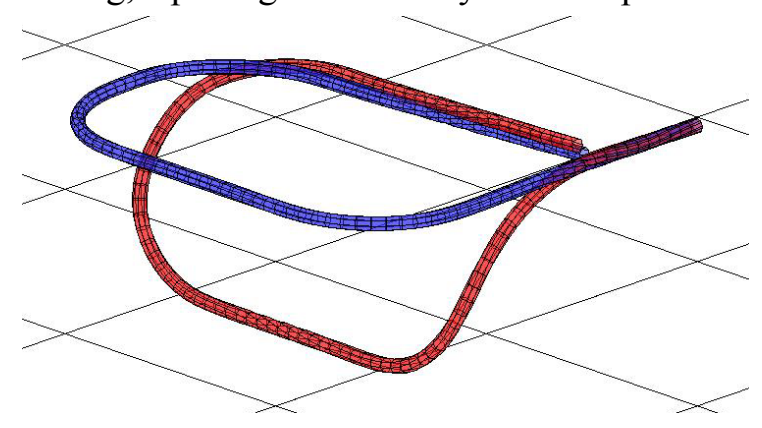

Figure 2: MCF shape measurements of a flexible surface. such as a photogrammetry. Temporarily applying retroreflective targets to the fiber and scanning the surface with a photogrammetric system can provide application engineers with a highly accurate threedimensional description of the MCF sensing cable layout. In theory, the MCF shape sensing system would be accurate enough that secondary verification of the layout scheme would not be necessary; however, unintentional externally induced twisting of the fiber during installation and local small-radius bending of the fiber can cause errors in the measurement. As such, independent measurement of the shape of the MCF fiber by a photogrammetric system can be utilized to determine where error sources exist in the fiber and error correction can ultimately be incorporated into subsequent MCF shape measurements.

\section{Conclusion}

Ground testing and verification of MCF shape measurement systems will be necessary as demand for in-flight measurement of deformable structures increases. While photogrammetry has an excellent track record of providing surface shape sensing in the aerospace testing environment, the in-flight implementation of the technology can prove difficult. It can, however, provide the independent measurements needed to quantify MCF shape measurement accuracy. Photogrammetric measurements can also provide a means of determining installation-related sources of MCF shape measurement error and can thus provide quantitative error compensation in MCF shape measurements.

\section{References}

[1] R. Duncan and M. Raum, "Characterization of a Fiber-Optic Shape and Position Sensor," SPIE International Symposium on Smart Structures and Materials, Proc. SPIE Vol. 6167, p. 616704, 2006.

[2] J.P. Moore and M.D. Rogge, "Shape sensing using multi-core fiber optic cable and parametric curve solutions," Optics Express, Vol. 20, Issue 3, pp. 2967-2973, 2012.

[3] M.E. Froggatt, J.W. Klein, D.K. Gifford and S.T. Kreger, “Optical Position and/or Shape Sensing," U.S. Patent Application, Pub. No. US 2011/0109898 A1, May 12, 2011.

[4] B.A. Childers, M.E. Froggatt, S.G. Allison, T.C. Moore, D.A. Hare, C.F. Batten and D.C. Jegley, "Use of 3000 Bragg grating strain sensors distributed on four eight-meter optical fibers during static load tests of a composite structure," Proc. SPIE Vol. 4332, pp. 133-142, 2001.

[5] R.S. Pappa, T.W. Jones, J.T. Black, A. Walford, S. Robson and M.R. Shortis, "Photogrammetry Methodology Development for Gossamer Spacecraft Structures," AIAA Paper 2002-1375, 2002. 